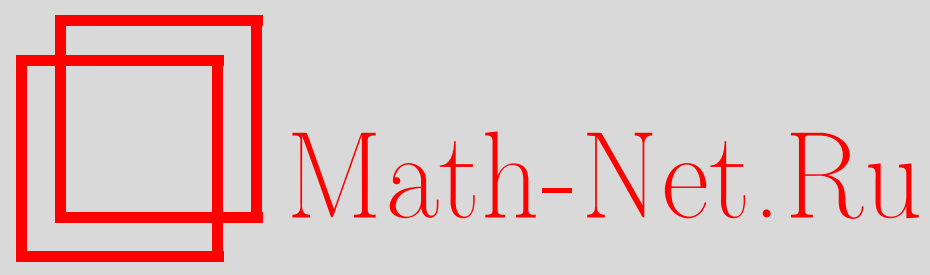

Н. Н. Фролов, Краевая задача для системы Бюргерса, $M a-$ тем. заметки, 1997, том 62, выпуск 6, 921-932

DOI: https://doi.org/10.4213/mzm1682

Использование Общероссийского математического портала Math-Net.Ru подразумевает, что вы прочитали и согласны с пользовательским соглашением http://www.mathnet.ru/rus/agreement

Параметры загрузки:

IP : 54.210 .77 .194

26 апреля 2023 г., 14:34:04

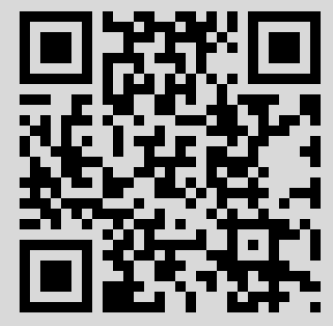


УДК 517.9

\section{КРАЕВАЯ ЗАДАЧА ДЛЯ СИСТЕМЫ БЮРГЕРСА}

\section{Н. Н. Фролов}

Рассматривается краевая задача

$$
\begin{gathered}
\operatorname{div}(\rho V)=0,\left.\quad \rho\right|_{\Gamma_{1}}=\rho_{0}, \\
\rho(V, \nabla V)=\nu \Delta V,\left.\quad V\right|_{\Gamma}=V^{0}
\end{gathered}
$$

относительно вектор-функции $V=\left(V_{1}, V_{2}\right)$ и скалярной функции $\rho \geqslant 0$ в прямоугольной области $\Omega \subset \mathbb{R}^{2}$ с границей $\Gamma$. Здесь

$$
\Gamma_{1}=\left\{x \in \Gamma:\left(V^{0}, n\right)<0\right\},\left.\quad V_{1}^{0}\right|_{\Gamma}>0, \quad \nu=\text { const }>0 .
$$

Доказьвается разрешимость данной задачи в классах Гёльдера.

Библиограффия: 8 названий.

1. Постановка задачи. Рассматривается стационарная задача, описывающая движение сжимаемой, неоднородной, вязкой жидкости в области $\Omega=(0, a) \times(0, b) \subset \mathbb{R}^{2}$ при постоянном давлении и отсутствии вектора массовых сил. Через $x=\left(x_{1}, x_{2}\right)$ обозначается точка области $\Omega$, через $\Gamma$ - граница $\Omega$, через $\Gamma_{j}(j=\overline{1,4})$ - части $\Gamma$, лежащие соответственно на прямых $x_{1}=0, x_{2}=0, x_{1}=a, x_{2}=b$. Задача описывается системой

$$
\begin{gathered}
\operatorname{div}(\rho V)=0,\left.\quad \rho\right|_{\Gamma_{1}}=\rho_{0}, \\
\rho(V, \nabla V)=\nu \Delta V,\left.\quad V\right|_{\Gamma}=V^{0}
\end{gathered}
$$

в $\Omega$ относительно неизвестных $\rho, V$. Здесь $V(x)=\left(V_{1}(x), V_{2}(x)\right)$ - вектор скорости, $\rho(x) \geqslant 0$ - плотность жидкости. Величины $V^{0}, \rho_{0}, \nu$ заданы: $V^{0}$ - вектор скорости жидкости на $\Gamma, \rho_{0} \geqslant 0$ - плотность на $\Gamma_{1}, \nu>0$ - коэффициент вязкости жидкости ( $\nu=$ const); $\Gamma_{1}, \Gamma_{3}$ - границы соответственно входа и выхода потока жидкости. Предполагается, что $V^{0}$ - непрерывная вектор-функция на $\Gamma$, удовлетворяющая условиям

$$
\begin{gathered}
\left.\left(V^{0}, n\right)\right|_{\Gamma_{1}}<0,\left.\quad\left(V^{0}, n\right)\right|_{\Gamma_{3}}>0,\left.\quad\left(V^{0}, n\right)\right|_{\Gamma_{2} \cup \Gamma_{4}}=0 \\
\left.\left(V^{0}, \tau\right)\right|_{\Gamma_{2}}>0,\left.\quad\left(V^{0}, \tau\right)\right|_{\Gamma_{4}}<0
\end{gathered}
$$

Работа выполнена при финансовой поддержке Российского фонда фундаментальных исследований, грант № 93-01-00277. 
где $n=\left(n_{1}, n_{2}\right)$ - вектор внешней нормали к $\Gamma, \tau$ - касательный вектор к $\Gamma$, определяющий положительную ориентацию на $\Gamma(n, \tau$ кусочно-постоянные на $\Gamma), \rho_{0} \geqslant 0\left(\rho_{0} \not \equiv 0\right)-$ непрерьвная функция на $\Gamma_{1}$.

В данной заметке устанавливается разрешимость задачи (1), (2) в пространствах Гёльдера для достаточно малых $\rho_{0}$ (см. (33)). Нестационарньй случай подобной задачи (где $x$ одномерно) рассмотрен в работах [1], [2].

2. Редукция задачи (1). Введем необходимые обозначения. Обозначим через $C^{l}(\bar{\Omega})\left(C^{0}(\bar{\Omega})=C(\bar{\Omega})\right)$ банахово пространство всех $l$ раз непрерьвно дифференцируемых (вектор-) функций на $\bar{\Omega}=\Omega \cup \Gamma$; через $C_{\alpha}^{l}(\bar{\Omega})$ - подпространство (вектор-) функций из $C^{l}(\bar{\Omega})$, производные $l$-го порядка которых удовлетворяют условию Гёльдера с показателем $\alpha(0<\alpha \leqslant 1), C_{\alpha}^{0}(\bar{\Omega})=C_{\alpha}(\bar{\Omega})$. Соответствующие пространства функций на $\Gamma$ обозначим через $C^{l}(\Gamma), C_{\alpha}^{l}(\Gamma)$.

Через $W_{p}^{l}(\Omega)(l \geqslant 0, p \geqslant 1)$ обозначим банахово пространство с нормой $\|\cdot\|_{p, l}$, состоящее из всех (вектор-) функций на $\Omega$, обобщенные производные которых до $l$-го порядка суммируемы на $\Omega$ со степенью $p ; L_{p}(\Omega)=W_{p}^{0}(\Omega), L_{\infty}(\Omega)$ - пространство существенно ограниченных (вектор-) функций на $\Omega$ (норму в $L_{p}, 1 \leqslant p \leqslant \infty$, обозначим через $\|\cdot\|_{p}$ ); $\stackrel{\circ}{W}_{p}^{l}(\Omega)$ - подпространство в $W_{p}^{l}(\Omega)$, в котором плотно множество $C_{0}^{l}(\Omega)$ всех финитных (вектор-) функций из $C^{l}(\bar{\Omega})$. Нормы во всех пространствах вводятся естественным образом (см. [3], [4]).

Пусть пара $(\rho, V)$ - решение задачи (1). Из первого уравнения (1) следует, что дифференциальная форма

$$
\alpha=-\rho V_{2} d x_{1}+\rho V_{1} d x_{2}
$$

замкнута в $\Omega: d \alpha=\operatorname{div}(\rho V) d x_{1} \wedge d x_{2}=0$. Отсюда в силу односвязности $\Omega$ вытекает, что $\alpha$ точна: $\alpha=d \varphi$. Таким образом, вектор-функция $\rho V \in W_{2}^{1}(\Omega)$ представима в виде $\rho V=\operatorname{rot} \varphi, \varphi \in W_{2}^{2}(\Omega)$, или

$$
\frac{\partial \varphi}{\partial x_{1}}=-\rho V_{2}, \quad \frac{\partial \varphi}{\partial x_{2}}=\rho V_{1}
$$

Подставляя эти соотношения в $(1)$, приходим к системе уравнений относительно неизвестных $(\varphi, V)$

$$
(V, \nabla \varphi)=0, \quad \nu \Delta V+[\varphi, V]=0
$$

где

$$
[\varphi, V]=\frac{\partial \varphi}{\partial x_{1}} \frac{\partial V}{\partial x_{2}}-\frac{\partial \varphi}{\partial x_{2}} \frac{\partial V}{\partial x_{1}}
$$

- скобка Пуассона функции $\varphi$ и вектор-функции $V$.

Далее, условие $\left.\operatorname{rot} \varphi\right|_{\Gamma_{1}}=\left.\rho V\right|_{\Gamma_{1}}=\left.\rho_{0} V^{0}\right|_{\Gamma_{1}}$ эквивалентно системе равенств

$$
\left.\frac{\partial \varphi}{\partial n}\right|_{\Gamma_{1}}=-\left.\rho_{0}\left(V^{0}, \tau\right)\right|_{\Gamma_{1}},\left.\quad \frac{\partial \varphi}{\partial \tau}\right|_{\Gamma_{1}}=\left.\rho_{0}\left(V^{0}, n\right)\right|_{\Gamma_{1}},
$$

которые однозначно (с точностью до аддитивной постоянной) определяют функцию $\varphi$ на $\Gamma_{1}$ :

$$
\left.\varphi\right|_{\Gamma_{1}}(x)=-\int_{\Gamma(0, x)} \rho_{0}\left(V^{0}, n\right) d s=\left.\int_{0}^{x_{2}} \rho_{0} V_{1}^{0}\right|_{\Gamma_{1}} d s \equiv \varphi_{0}\left(x_{2}\right) .
$$


Здесь $\Gamma(0, x)$ - часть отрезка $\Gamma_{1}$ с началом в точке $x=0$ и концом в точке $x=\left(0, x_{2}\right) \in \Gamma_{1}$. Таким образом, задача $(1),(2)$ свелась к поиску пары $(\varphi, V)$, удовлетворяющей уравнениям (4) в $\Omega$ и граничным условиям

$$
\left.V\right|_{\Gamma}=V^{0},\left.\quad \varphi\right|_{\Gamma_{1}}=\varphi_{0}
$$

Обратно, если пара $(\varphi, V)$ - гладкое решение задачи $(4),(6)$, где $\varphi_{0}$ имеет вид (5), то из первого уравнения (4) вытекает существование функции $\rho$, для которой вьполнены равенства (3), и, следовательно, справедливо первое уравнение (1). Далее, подставляя (3) во второе уравнение (4), приходим к уравнению (1) для $V$. Из (3), (5), (6) вытекает вьполнение граничных условий (1) для $V$ и $\rho$ :

$$
\left.\rho V_{1}\right|_{\Gamma_{1}}=\left.\frac{\partial \varphi}{\partial x_{2}}\right|_{\Gamma_{1}}=\left.\frac{\partial \varphi_{0}}{\partial x_{2}}\right|_{\Gamma_{1}}=\left.\rho_{0} V_{1}^{0} \Longrightarrow \rho\right|_{\Gamma_{1}}=\rho_{0} .
$$

Как будет показано (см. п. 3), в условиях (2) $\partial \varphi / \partial x_{2} \geqslant 0$. Следовательно, согласно (3) плотность жидкости на выходе $\Gamma_{3}$ равна

$$
\left.\rho\right|_{\Gamma_{3}}=\left.\left(\frac{1}{V_{1}^{0}} \frac{\partial \varphi}{\partial x_{2}}\right)\right|_{\Gamma_{3}} \geqslant 0 .
$$

3. Задача Коши для функции тока. Рассмотрим задачу Коши относительно функции $\varphi$ :

$$
(V, \nabla \varphi)=0, \quad x \in \Omega,\left.\quad \varphi\right|_{\Gamma_{1}}=\varphi_{0} .
$$

Нам потребуются некоторые оценки для решений данной задачи. Предварительно докажем следующее утверждение.

Лемма 1. Пусть $f \in W_{2}^{2}(\Omega),\left.f\right|_{\Gamma_{2} \cup \Gamma_{4}}=0$. Тогда через каждую точку $x \in \bar{\Omega}$ проходит единственная интегральная кривая уравнения

$$
\frac{d \xi}{d t}=f(t, \xi), \quad 0 \leqslant t \leqslant a
$$

лежащая в $\bar{\Omega}$. При әтом $\xi(t, x) \in C^{1}([0, a] \times \bar{\Omega})$ и справедливы равенства

$$
\begin{gathered}
\frac{\partial \xi(t, x)}{\partial x_{1}}=-f(x) \frac{\partial \xi(t, x)}{\partial x_{2}}, \quad \frac{\partial \xi(t, x)}{\partial x_{2}}=\exp \{-I(t, x)\}, \\
I(t, x)=\int_{t}^{x_{1}} \frac{\partial f}{\partial x_{2}}(s, \xi(s, x)) d s .
\end{gathered}
$$

ДокАЗАТЕЛЬСТво. В случае, когда $f \in C^{1}(\bar{\Omega}),\left.f\right|_{\Gamma_{2} \cup \Gamma_{4}}=0$, утверждения леммы 1 общеизвестны (см. [5, с. 92]). Пусть $f \in W_{2}^{2}(\Omega)$. Существование интегральной кривой уравнения (8), проходящей через точку $x \in \bar{\Omega}$ и лежащей в $\bar{\Omega}$, вытекает из условий $W_{2}^{2}(\Omega) \subset C_{\alpha}(\bar{\Omega})(0<\alpha<1$ любое $),\left.f\right|_{\Gamma_{2} \cup \Gamma_{4}}=0$. Далее, согласно теореме вложения (см. [6]) для таких $f$

$$
\left|f\left(x_{1}, x_{2}\right)-f\left(x_{1}, x_{2}^{\prime}\right)\right| \leqslant c\|f\|_{2,2} \cdot\left|x_{2}-x_{2}^{\prime}\right|\left(1+|\ln | x_{2}-x_{2}^{\prime}||^{1 / 2}\right)
$$


где $x_{1} \in[0, a], x_{2}, x_{2}^{\prime} \in[0, b]-$ произвольные точки, а постоянная $c$ зависит лишь от $\Omega$. Из этой оценки по теореме Осгуда (см. [5, с. 51]) следует единственность решения задачи Коши (8).

Покажем, что $\xi(t, x) \in C^{1}([0, a] \times \bar{\Omega})$ и справедливы равенства (9). Для этого рассмотрим последовательность $f_{n} \in C^{2}(\bar{\Omega}),\left.f_{n}\right|_{\Gamma_{2} \cup \Gamma_{4}}=0$, такую, что $\left\|f_{n}-f\right\|_{2,2} \rightarrow 0$ $(n \rightarrow \infty)$. Пусть $\xi_{n}(t, x)$ - интегральная кривая уравнения $(8)$, в котором $f=f_{n}$. Поскольку $f_{n} \rightarrow f$ в $C(\bar{\Omega})$, то (см. $[5$, с. 80$\left.]\right) \xi_{n}(t, x) \rightarrow \xi(t, x)$ в $C([0, a] \times \bar{\Omega})$ при $n \rightarrow \infty$ и, следовательно, $\xi(t, x), \partial \xi / \partial t \in C([0, a] \times \bar{\Omega})$.

Рассмотрим последовательности

$$
\begin{gathered}
\frac{\partial \xi_{n}(t, x)}{\partial x_{1}}=-f_{n}(x) \frac{\partial \xi_{n}(t, x)}{\partial x_{2}}, \quad \frac{\partial \xi_{n}(t, x)}{\partial x_{2}}=\exp \left\{-I_{n}(t, x)\right\} \\
I_{n}(t, x)=\int_{t}^{x_{1}} \frac{\partial f_{n}}{\partial x_{2}}\left(s, \xi_{n}(s, x)\right) d s
\end{gathered}
$$

Покажем, что они сходятся в $C([0, a] \times \bar{\Omega})$ при $n \rightarrow \infty$ к соответствуюшим выражениям в правых частях (9). Поскольку $f_{n} \rightarrow f$ в $C(\bar{\Omega})$, достаточно установить, что $\partial \xi_{n} / \partial x_{2} \rightarrow$ $\partial \xi / \partial x_{2}$ в $C([0, a] \times \bar{\Omega})$.

Из теорем о следах функций $\psi \in W_{2}^{1}(\Omega)$ вытекают неравенства

$$
\begin{gathered}
\int_{t}^{x_{1}}|\psi(s, \eta(s))| d s \leqslant c_{0} \int_{\Omega}\left(\left|\frac{\partial \psi}{\partial x_{2}}\right|+|\psi|\right) d x \\
\int_{t}^{x_{1}}|\psi(s, \eta(s))-\psi(s, \xi(s))| d s \leqslant\left(\int_{\Omega}\left|\frac{\partial \psi}{\partial x_{2}}\right|^{2} d x \int_{0}^{a}|\eta(s)-\xi(s)| d s\right)^{1 / 2}
\end{gathered}
$$

справедливые для любых $t, x_{1} \in[0, a]$ и функций $\eta(s), \xi(s) \in C\left[t, x_{1}\right], 0 \leqslant \eta, \xi \leqslant b, \mathrm{c}$ постоянной $c_{0}$, зависящей лишь от $\Omega\left(c_{0} \leqslant \max (8,11 / b)\right)$. Используя оценку $(10)$ при

$$
\psi=\frac{\partial\left(f_{n}-f\right)}{\partial x_{2}}, \quad \eta=\xi_{n}(s, x),
$$

оценку (11) при

$$
\psi=\frac{\partial f}{\partial x_{2}}, \quad \eta=\xi_{n}(s, x), \quad \xi=\xi(s, x),
$$

получаем, что равномерно по $x \in \bar{\Omega}, t \in[0, a]$

$$
\begin{aligned}
\left|I_{n}(t, x)-I(t, x)\right| \leqslant & c_{0} \int_{\Omega}\left(\left|\frac{\partial^{2}\left(f_{n}-f\right)}{\partial x_{2}^{2}}\right|+\left|\frac{\partial\left(f_{n}-f\right)}{\partial x_{2}}\right|\right) d x \\
& +\left(\int_{\Omega}\left|\frac{\partial^{2} f}{\partial x_{2}^{2}}\right|^{2} d x \int_{0}^{a}\left|\xi_{n}(s, x)-\xi(s, x)\right| d s\right)^{1 / 2} \rightarrow 0, \quad n \rightarrow \infty
\end{aligned}
$$

Отсюда следует, что $\partial \xi_{n} / \partial x_{j} \rightarrow \partial \xi / \partial x_{j}(j=1,2, n \rightarrow \infty)$ в $C([0, a] \times \bar{\Omega})$. Здесь $\partial \xi / \partial x_{j}$ - выражения, стоящие в правьх частях (9). Лемма доказана.

Аналогично устанавливается, что из сходимости $\left\|f_{n}-f\right\|_{2,2} \rightarrow 0\left(f_{n}, f \in W_{2}^{2}(\Omega)\right)$ вытекает сходимость $\xi_{n}(t, x) \rightarrow \xi(t, x)$ в $C^{1}([0, a] \times \bar{\Omega})$. 
ПРЕДЛОЖЕНИЕ. Пусть $\varphi_{0} \in C^{1}\left(\Gamma_{1}\right)$,

$$
V \in \mathscr{K}(\Omega) \equiv\left\{V \in W_{2}^{2}(\Omega): V_{1}>0,\left.V_{2}\right|_{\Gamma_{2} \cup \Gamma_{4}}=0\right\} .
$$

Тогда в $C^{1}(\bar{\Omega})$ существует единственное решение $\varphi=\varphi(V, x)$ задачи $(7)$, причем

$$
\|\varphi(V, \cdot)\|_{C^{1}} \leqslant\left\|\varphi_{0}\right\|_{\infty}+\left\|\varphi_{0}^{\prime}\right\|_{\infty} \frac{\|V\|_{\infty}}{\min _{\Gamma_{1}} V_{1}} \exp \left\{\frac{2 c_{0} \sqrt{a b}}{\min _{\Omega} V_{1}}\|V\|_{2,2}\right\},
$$

где с $c_{0}$ - постоянная вложения из (10) и оператор $V \mapsto \varphi(V, \cdot), \mathscr{K}(\Omega) \rightarrow C^{1}(\bar{\Omega})$ непрерывен.

$E c \Omega u V \in \mathscr{K}(\Omega) \cap C_{\beta}^{1}(\bar{\Omega}), \varphi_{0} \in C_{\delta}^{1}\left(\Gamma_{1}\right), \operatorname{mo} \varphi \in C_{\alpha}^{1}(\bar{\Omega}) u$

$$
\|\varphi(V, \cdot)\|_{C_{\alpha}^{1}} \leqslant \sigma\left(\frac{1}{\min _{\Omega} V_{1}},\|V\|_{C_{\alpha}^{1}}\right), \quad \alpha=\min (\beta, \delta),
$$

где $\sigma(t, \tau)$ - возрастающая функиия по каждой из переменных $t>0, \tau \geqslant 0$.

ДокАЗАТЕльСтво. Поскольку $V_{1}>0$ на $\bar{\Omega}$, то уравнение (7) эквивалентно уравнению

$$
\frac{\partial \varphi}{\partial x_{1}}+f \frac{\partial \varphi}{\partial x_{2}}=0, \quad \text { где } f=\frac{V_{2}}{V_{1}} \in W_{2}^{2}(\Omega),\left.\quad f\right|_{\Gamma_{2} \cup \Gamma_{4}}=0 .
$$

Пусть $\xi_{V}(t, x) \equiv \xi(t, x)$ - интегральная кривая уравнения (8) ( $f$ определена из (14)), проходящая через точку $x \in \bar{\Omega}$. Из леммы 1 вытекает, что на $\bar{\Omega}$ определена функция $\xi(0, x) \in C^{1}(\bar{\Omega})$, причем

$$
\frac{\partial \xi(0, x)}{\partial x_{1}}=-f(x) \frac{\partial \xi(0, x)}{\partial x_{2}}, \quad \frac{\partial \xi(0, x)}{\partial x_{2}}=\exp \{-I(0, x)\} .
$$

Отсюда получаем, что функция

$$
\varphi(x)=\varphi_{0}(\xi(0, x)) \in C^{1}(\bar{\Omega})
$$

является решением задачи Коши (7), причем

$$
\frac{\partial \varphi}{\partial x_{1}}=-f(x) \varphi_{0}^{\prime}(\xi(0, x)) \frac{\partial \xi(0, x)}{\partial x_{2}}, \quad \frac{\partial \varphi}{\partial x_{2}}=\varphi_{0}^{\prime}(\xi(0, x)) \frac{\partial \xi(0, x)}{\partial x_{2}} .
$$

В частности, если $\varphi_{0}$ определено равенством (5), то из (15), (17) следует, что $\partial \varphi / \partial x_{2} \geqslant 0$. Установим оценку (12). Полагая в неравенстве (10) $\psi=\operatorname{div} V, \eta=\xi(s, x)$, находим

$$
\left|\int_{0}^{x_{1}} \frac{\operatorname{div} V}{V_{1}}(s, \xi(s, x)) d s\right| \leqslant \frac{1}{\min _{\Omega} V_{1}} \int_{0}^{x_{1}}|\operatorname{div} V(s, \xi(s, x))| d s \leqslant \frac{2 c_{0} \sqrt{a b}}{\min _{\Omega} V_{1}}\|V\|_{2,2} .
$$

Далее, из равенства

$$
\operatorname{div} V(s, \xi(s, x))=\frac{d}{d s} V_{1}(s, \xi(s, x))+V_{1}(s, \xi(s, x)) \frac{\partial f}{\partial x_{2}}(s, \xi(s, x))
$$


получаем

$$
I(0, x)=\int_{0}^{x_{1}} \frac{\operatorname{div} V}{V_{1}}(s, \xi(s, x)) d s-\ln \frac{V_{1}(x)}{V_{1}(0, \xi(0, x))}
$$

и, следовательно, согласно (18)

$$
\left|\frac{\partial \xi(0, x)}{\partial x_{2}}\right| \leqslant \frac{V_{1}(x)}{V_{1}(0, \xi(0, x))} \exp \left\{\frac{2 c_{0} \sqrt{a b}}{\min _{\Omega} V_{1}}\|V\|_{2,2}\right\} .
$$

Из (15)-(17) и последней оценки вытекает (12).

Пусть $V, V_{n} \in \mathscr{K}(\Omega)$ и $\left\|V_{n}-V\right\|_{2,2} \rightarrow 0$. Тогда $\left\|f_{n}-f\right\|_{2,2} \rightarrow 0(n \rightarrow \infty)$ и, как уже отмечалось, $\xi_{V_{n}}(0, x) \rightarrow \xi_{V}(0, x)$ в $C^{1}(\bar{\Omega})$. Поэтому из $(16),(17)$ следует $\varphi\left(V_{n}, \cdot\right) \rightarrow \varphi(V, \cdot)$ в $C^{1}(\bar{\Omega})$, т.е. оператор $V \rightarrow \varphi(V, \cdot)$ непрерьвен.

Установим теперь оценку (13). Обозначим через $|\psi|_{\alpha}$ константу Гёльдера функции $\psi \in C_{\alpha}(\bar{\Omega})$. При помощи неравенства

$$
|\xi(t, x+h)-\xi(t, x)| \leqslant|h| \sqrt{1+\|f\|_{\infty}^{2}} \exp \left\{a\left\|\frac{\partial f}{\partial x_{2}}\right\|_{\infty}\right\}, \quad x, x+h \in \Omega,
$$

из (17), непосредственно вычисляя, находим

$$
\begin{aligned}
&\left|\frac{\partial \varphi}{\partial x_{2}}\right|_{\alpha} \leqslant\left|\varphi_{0}^{\prime}\right|_{\alpha}(1+\left.\|f\|_{\infty}^{2}\right)^{\alpha / 2} \exp \left\{a(\alpha+1)\left\|\frac{\partial f}{\partial x_{2}}\right\|_{\infty}\right\}+\left\|\varphi_{0}^{\prime}\right\|_{\infty} \cdot|\exp \{-I(0, x)\}|_{\alpha}, \\
&|\exp \{-I(0, x)\}|_{\alpha} \leqslant\left(a^{1-\alpha}\left\|\frac{\partial f}{\partial x_{2}}\right\|_{\infty}+a\left|\frac{\partial f}{\partial x_{2}}\right|_{\alpha}\left(1+\|f\|_{\infty}^{2}\right)^{\alpha / 2} \exp \left\{\alpha a\left\|\frac{\partial f}{\partial x_{2}}\right\|_{\infty}\right\}\right) \\
& \times \exp \left\{a\left\|\frac{\partial f}{\partial x_{2}}\right\|_{\infty}\right\}, \\
&\left|\frac{\partial \varphi}{\partial x_{1}}\right|_{\alpha} \leqslant D^{1-\alpha}\|\nabla f\|_{\infty} \cdot\left\|\frac{\partial \varphi}{\partial x_{2}}\right\|_{\infty}+\|f\|_{\infty} \cdot\left|\frac{\partial \varphi}{\partial x_{2}}\right|_{\alpha},
\end{aligned}
$$

где $D$ - диаметр $\Omega$. Из этих неравенств с учетом $f=V_{2} / V_{1}$ и (12) вытекает (13). Предложение доказано.

4. Краевая задача для вектора скорости. Рассмотрим краевую задачу

$$
\nu \Delta V+[\varphi, V]=0, \quad x \in \Omega,\left.\quad V\right|_{\Gamma}=\left.V^{0}\right|_{\Gamma}
$$

относительно вектор-функции $V$. Нам потребуются некоторые оценки для решений задачи (19).

Лемма 2. Пусть $\varphi \in C^{1}(\bar{\Omega}), V^{0} \in W_{p}^{1}(\Omega)(p \geqslant 2)$. Тогда в $W_{p}^{1}(\Omega)$ существует единственное решение $V$ задачи (19). При әтом справедливы оценки:

a) $п р и$ u $=2$

$$
\left\|\nabla\left(V-V^{0}\right)\right\|_{2} \leqslant\left\|\nabla V^{0}\right\|_{2}\left(1+\frac{\gamma}{\nu}\|\nabla \varphi\|_{\infty}\right), \quad \gamma=\min (a, b) ;
$$

б) при $p>2$

$$
\min _{\Gamma} V_{j}^{0} \leqslant V_{j}(x) \leqslant \max _{\Gamma} V_{j}^{0}, \quad j=1,2, \quad x \in \bar{\Omega} .
$$


ДокАЗАТЕльство. Существование и единственность решения задачи $(19)$ в $W_{p}^{1}(\Omega)$ хорошо известно (см. [4], [7]). Оценка (20) вытекает из интегрального тождества

$$
\nu \int_{\Omega} \nabla u \cdot \nabla w d x=\int_{\Omega}\left\{[\varphi, V] w-\nu \nabla V^{0} \cdot \nabla w\right\} d x, \quad w \in \stackrel{\circ}{W}_{2}^{1}(\Omega),
$$

определяющего решение $V=u+V^{0}$ задачи $(19)$ в $W_{2}^{1}(\Omega)$, если положить в нем $w=u$, оценить правую часть с помощью неравенства Гёльдера и воспользоваться неравенством Фридрихса $\|u\|_{2} \leqslant \gamma\|\nabla u\|_{2}\left(u \in \stackrel{\circ}{W_{2}^{1}}(\Omega)\right)$.

Для доказательства неравенств $(21)$ возьмем произвольные $\varepsilon>0, x_{0} \in \Omega$ и подберем $\delta(0<2 \delta<\gamma)$ так, чтобы

$$
\left|V(y)-V^{0}(x)\right|<\varepsilon \quad \text { при }|x-y|<\sqrt{2} \delta, \quad x \in \Gamma, \quad y \in \bar{\Omega} .
$$

Положим $\Omega_{\delta}=(\delta, a-\delta) \times(\delta, b-\delta)$. Можно считать, что $x_{0} \in \bar{\Omega}_{\delta}$. Обозначим через $V_{h}=V * \omega_{h}(0<h<\delta)$ усреднение функции $V$ стандартным ядром $\omega_{h}$ :

$$
\begin{aligned}
& \omega_{h}(|x|)=c_{h} \exp \left\{-\frac{h^{2}}{h^{2}-|x|^{2}}\right\} \quad \text { при }|x|<h, \\
& \omega_{h}=0 \quad \text { при }|x| \geqslant h, \quad \int_{\mathbb{R}^{2}} \omega_{h}(|x|) d x=1 .
\end{aligned}
$$

Тогда для решения $V \in W_{p}^{1}(\Omega) \subset C_{\alpha}(\bar{\Omega})(\alpha=1-2 / p, p>2)$ справедливо соотношение

$$
\left\|V_{h}-V\right\|_{C\left(\bar{\Omega}_{\delta}\right)} \rightarrow 0, \quad h \rightarrow 0 .
$$

Положим в $(22) w=w_{h}$, где $w \in C_{0}^{1}(\Omega)$ и $\operatorname{supp} w \subset \bar{\Omega}_{\delta}$. Учитывая, что $\nabla w_{h}=(\nabla w)_{h}$, после интегрирования по частям левой части $(22)$, получим

$$
\int_{\Omega}\left\{\nu \Delta V_{h}+[\varphi, V]_{h}\right\} w d x=0, \quad w \in C_{0}^{1}(\Omega) .
$$

Отсюда вытекает, что функция $V_{h} \in C^{2}\left(\bar{\Omega}_{\delta}\right)$ является классическим решением уравнения

$$
\nu \Delta V_{h}(x)+\left[\varphi, V_{h}\right](x)=f_{h}(x), \quad x \in \Omega_{\delta},
$$

где $f_{h}=\left[\varphi, V_{h}\right]-[\varphi, V]_{h} \in C(\bar{\Omega})$. Следовательно, по принципу максимума

$$
\begin{gathered}
\min \left(-c\left\|\left(f_{j}\right)_{h}\right\|_{C\left(\bar{\Omega}_{\delta}\right)}, \min _{\Gamma_{\delta}}\left(V_{j}\right)_{h}\right) \leqslant\left(V_{j}\right)_{h}(x) \leqslant \max \left(c\left\|\left(f_{j}\right)_{h}\right\|_{C\left(\bar{\Omega}_{\delta}\right)}, \max _{\Gamma_{\delta}}\left(V_{j}\right)_{h}\right), \\
x \in \bar{\Omega}_{\delta}, \quad j=1,2,
\end{gathered}
$$

где $\Gamma_{\delta}-$ гранища области $\Omega_{\delta}, c>0$ - постоянная, зависящая лишњ от $\nu$ и любого из $\left\|\partial \varphi / \partial x_{j}\right\|_{C(\bar{\Omega})}(j=1,2)$. Переходя к пределу при $h \rightarrow 0$, согласно $(24)$ получаем оценки

$$
\min _{\Gamma_{\delta}} V_{j} \leqslant V_{j}(x) \leqslant \max _{\Gamma_{\delta}} V_{j}, \quad x \in \bar{\Omega}_{\delta}, \quad j=1,2 .
$$

Отсюда, используя (23), получаем

$$
\min _{\Gamma} V_{j}^{0}-\varepsilon \leqslant V_{j}\left(x_{0}\right) \leqslant \max _{\Gamma} V_{j}^{0}+\varepsilon .
$$

Последние оценки в силу произвольности $x_{0} \in \Omega, \varepsilon>0$ приводят к неравенствам (21). Лемма доказана. 
Лемма 3. Пусть $\varphi, \varphi_{j} \in C^{1}(\bar{\Omega})(j=1,2), V^{0} \in W_{p}^{2}(\Omega)(p \geqslant 2)$. Тогда решение $V=V(\varphi, x)$ задачи (19) принадлежит классу $W_{p}^{2}(\Omega)$ и справедливы оченки

$$
\begin{gathered}
\|V(\varphi, \cdot)\|_{2,2} \leqslant\left\|V^{0}\right\|_{2,2}+c_{2}\left\|\Delta V^{0}\right\|_{2}+\frac{c_{2}}{\nu}\left\|\nabla V^{0}\right\|_{2}\left(2+\frac{\gamma}{\nu}\|\nabla \varphi\|_{\infty}\right)\|\nabla \varphi\|_{\infty}, \\
\left\|V\left(\varphi_{1}, \cdot\right)-V\left(\varphi_{2}, \cdot\right)\right\|_{2,2} \leqslant \frac{c_{2}}{\nu}\left\|\nabla V\left(\varphi_{2}, \cdot\right)\right\|_{2}\left(1+\frac{\gamma}{\nu}\left\|\nabla \varphi_{1}\right\|_{\infty}\right)\left\|\nabla\left(\varphi_{1}-\varphi_{2}\right)\right\|_{\infty}, \\
\|V(\varphi, \cdot)\|_{p, 2} \leqslant c\left(1+\|\nabla \varphi\|_{\infty}\right)^{3}
\end{gathered}
$$

где $c_{2}=\left\|\Delta^{-1}\right\|-$ норма оператора, обратного $\kappa \Delta: W_{2}^{2} \cap \stackrel{\circ}{W_{2}^{1}} \rightarrow L_{2}$, а с зависит лишь от $p, \nu, \Omega u\left\|V^{0}\right\|_{p, 2}$.

ДокАЗАТЕльСтво. Принадлежность решения $V=u+V^{0}$ к $W_{p}^{2}(\Omega)$ вытекает из [7]. Для доказательства оценки (25) запишем задачу (19) в виде

$$
\Delta u=f,\left.\quad u\right|_{\Gamma}=0, \quad \text { где } f=-\Delta V^{0}-\frac{1}{\nu}\left[\varphi, u+V^{0}\right],
$$

и воспользуемся неравенством $\|u\|_{2,2} \leqslant c_{2}\|f\|_{2}$. Оценивая $\|f\|_{2}$ по неравенству Гёльдера и используя (20), получим

$$
\|u\|_{2,2} \leqslant c_{2}\left\|\Delta V^{0}\right\|_{2}+\frac{c_{2}}{\nu}\left\|\nabla V^{0}\right\|_{2}\left(2+\frac{\gamma}{\nu}\|\nabla \varphi\|_{\infty}\right)\|\nabla \varphi\|_{\infty}
$$

откуда следует (25).

Для доказательства (26) заметим, что разность $w=V\left(\varphi_{1}, \cdot\right)-V\left(\varphi_{2}, \cdot\right)-$ решение задачи

$$
\Delta w=f_{1},\left.\quad w\right|_{\Gamma}=0, \quad \text { где } f_{1}=\frac{1}{\nu}\left[\varphi_{2}-\varphi_{1}, V\left(\varphi_{2}, \cdot\right)\right]-\frac{1}{\nu}\left[\varphi_{1}, w\right] .
$$

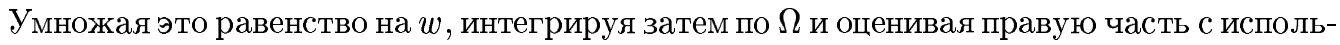
зованием неравенства Фридрихса, находим, что

$$
\|\nabla w\|_{2} \leqslant \frac{\gamma}{\nu}\left\|\nabla V\left(\varphi_{2}, \cdot\right)\right\|_{2} \cdot\left\|\nabla\left(\varphi_{2}-\varphi_{1}\right)\right\|_{\infty}
$$

Используя это соотношение, оценим $\left\|f_{1}\right\|_{2}$ с помощью неравенства Гёльдера. Подставив полученную оценку в неравенство $\|w\|_{2,2} \leqslant c_{2}\left\|f_{1}\right\|_{2}$, получим (26).

Для доказательства (27) воспользуемся неравенством $\|u\|_{p, 2} \leqslant c_{p}\|f\|_{p}$, где $u$ и $f$ из (28). Тогда

$$
\|u\|_{p, 2} \leqslant c_{p}\left\|\Delta V^{0}\right\|_{p}+\frac{c_{p}}{\nu}\left(\left\|\nabla V^{0}\right\|_{p}+\|\nabla u\|_{p}\right)\|\nabla \varphi\|_{\infty} .
$$

Выражение $\|\nabla u\|_{p}$ согласно вложению $W_{2}^{2}(\Omega) \subset W_{p}^{1}(\Omega)$ оценивается правой частью $(29)$ : $\|\nabla u\|_{p} \leqslant c_{p}^{\prime}\|u\|_{2,2}$. Поэтому для $\|u\|_{p, 2}$ и, следовательно, для $\|V\|_{p, 2}$ справедливо неравенство (27). Лемма доказана.

Из (25), (26) вытекает непрерьвность оператора $\varphi \mapsto V(\varphi, \cdot), C^{1}(\bar{\Omega}) \rightarrow W_{2}^{2}(\Omega)$, где $V(\varphi, x)$ - решение в $W_{2}^{2}(\Omega)$ задачи $(19)$ с фиксированным $V^{0} \in W_{2}^{2}(\Omega)$. 
5. Существование решений задачи $(2),(4),(6)$. Пусть $\varphi_{0} \in C^{1}\left(\Gamma_{1}\right), V^{0} \in W_{2}^{2}(\Omega)$ и $V_{1}^{0}>0$ на $\Gamma$. Рассмотрим в $C^{1}(\bar{\Omega})$ оператор $A \varphi=\varphi_{0}\left(\xi_{V(\varphi, \cdot)}(0, x)\right)$, где $V(\varphi, \cdot)$ решение задачи (19), а $\xi_{V}$-интегральная кривая уравнения (8) (в котором $f=V_{2}(\varphi, \cdot)$ / $\left.V_{1}(\varphi, \cdot)\right)$, проходящая через точку $x \in \bar{\Omega}$. Из предложения и леммы 2 вытекает, что

$$
\|A \varphi\|_{C^{1}} \leqslant\left\|\varphi_{0}\right\|_{\infty}+\left\|\varphi_{0}^{\prime}\right\|_{\infty} \frac{\sqrt{2} \max _{\Gamma}\left|V^{0}\right|}{\min _{\Gamma} V_{1}^{0}} \exp \left\{\frac{2 c_{0} \sqrt{a b}}{\min _{\Gamma} V_{1}^{0}}\|V(\varphi, \cdot)\|_{2,2}\right\} .
$$

Подставляя в это неравенство оценку (25) из леммы 3 , получаем

$$
\|A \varphi\|_{C^{1}} \leqslant c+d \exp \left\{\alpha t+\beta t^{2}\right\}=\psi(t), \quad t=\|\varphi\|_{C^{1}} .
$$

Здесь использованы обозначения

$$
\begin{gathered}
c=\left\|\varphi_{0}\right\|_{\infty}, \quad d=\left\|\varphi_{0}^{\prime}\right\|_{\infty} \frac{\sqrt{2} \max _{\Gamma}\left|V^{0}\right|}{\min _{\Gamma} V_{1}^{0}} \exp \left\{\sigma\left(\left\|V^{0}\right\|_{2,2}+c_{2}\left\|\Delta V^{0}\right\|_{2}\right)\right\}, \\
\sigma=\frac{2 c_{0} \sqrt{a b}}{\min _{\Gamma} V_{1}^{0}}, \quad \alpha=\sigma \frac{2 c_{2}}{\nu}\left\|\nabla V^{0}\right\|_{2}, \quad \beta=\frac{\gamma}{2 \nu} \alpha .
\end{gathered}
$$

Теорема 1. Пусть $V^{0} \in W_{p}^{2}(\Omega)(p>2), \varphi_{0} \in C_{\delta}^{1}\left(\Gamma_{1}\right)$, выполнены условия (2) $и$

$$
d e^{K} \sqrt{\alpha^{2}+4 \beta K} \leqslant 1, \quad K=\frac{1}{2}\left(1+\sqrt{1+\frac{4 c}{d e}}\right) .
$$

Тогда существует решение $(\varphi, V)$ задачи (4), (6), причем $\varphi \in C_{\delta^{\prime}}^{1}(\bar{\Omega}), V \in W_{p}^{2}(\Omega)$, $\delta^{\prime}=\min (\delta, 1-2 / p)$.

ДокАЗАТЕльСтво. Если $\varphi \in C^{1}(\bar{\Omega})$ является неподвижной точкой оператора $A$, то согласно предложению и лемме 3 пара $(\varphi, V(\varphi, \cdot))$ - решение задачи $(4),(6)$, причем $V=V(\varphi, \cdot) \in W_{p}^{2}(\Omega)$.

Пусть $t_{0}>0$ - корень уравнения $\left(\psi(t) t^{-1}\right)^{\prime}=0$. Нетрудно показать, что

$$
t_{0} \leqslant t_{1} \equiv \frac{1}{2 \beta}\left(\sqrt{\alpha^{2}+4 \beta K}-\alpha\right),
$$

где $K$ определено равенством (32). Если для $c, d, \alpha, \beta$ из (31) выполнено условие $\psi\left(t_{0}\right) \times$ $t_{0}^{-1} \leqslant 1$, то существует корень $R\left(0<R \leqslant t_{0}\right)$ уравнения $\psi(t)=t$. Последнее условие вытекает из (32), так как

$\psi\left(t_{0}\right) t_{0}^{-1}=d\left(\alpha+2 \beta t_{0}\right) \exp \left\{\alpha t_{0}+\beta t_{0}^{2}\right\} \leqslant d\left(\alpha+2 \beta t_{1}\right) \exp \left\{\alpha t_{1}+\beta t_{1}^{2}\right\}=d e^{K} \sqrt{\alpha^{2}+4 \beta K}$.

Таким образом, согласно (30) оператор $A: C^{1}(\bar{\Omega}) \rightarrow C^{1}(\bar{\Omega})$ отображает шар $\|\varphi\|_{C^{1}} \leqslant R$ в себя. Этот оператор непрерывен, что следует из непрерывности оператора $\varphi \mapsto V(\varphi, \cdot)$, $C^{1}(\bar{\Omega}) \rightarrow W_{2}^{2}(\Omega)$ (лемма 3) и непрерьвности оператора $V \mapsto \varphi(V, \cdot), \mathscr{K}(\Omega) \rightarrow C^{1}(\bar{\Omega})$ (предложение). Покажем, что $A$ действует из $C^{1}(\bar{\Omega})$ в $C_{\delta^{\prime}}^{1}(\bar{\Omega})$ и ограничен. 
Действительно, по лемме 3 оператор $\varphi \mapsto V(\varphi, \cdot), C^{1}(\bar{\Omega}) \rightarrow W_{p}^{2}(\Omega)$ ограничен. Если $p>2$, то он ограничен из $C^{1}(\bar{\Omega})$ в $C_{\alpha^{\prime}}^{1}(\bar{\Omega})\left(\alpha^{\prime}=1-2 / p\right)$. Кроме того, по предложению оператор $V \mapsto \varphi(V, \cdot), \mathscr{K}(\Omega) \cap C_{\alpha^{\prime}}^{1}(\bar{\Omega}) \rightarrow C_{\delta^{\prime}}^{1}(\bar{\Omega})$ ограничен, $\delta^{\prime}=\min \left(\alpha^{\prime}, \delta\right)$.

В силу компактности вложения $C_{\delta^{\prime}}^{1}(\bar{\Omega}) \subset C^{1}(\bar{\Omega}) \quad A$ вполне непрерьвен в $C^{1}(\bar{\Omega})$, и, следовательно, по теореме Шаудера в шаре $\|\varphi\|_{C^{1}} \leqslant R$ существует неподвижная точка $\varphi$ оператора $A$. Очевидно, $\varphi \in C_{\delta^{\prime}}^{1}(\bar{\Omega})$. Теорема доказана.

Пусть $\varphi_{0}$ имеет вид (5). Условие (32) с учетом (31) можно переписать в виде

$$
\begin{aligned}
\max _{\Gamma_{1}} \rho_{0} V_{1}^{0} \leqslant & e^{-K}\left(\alpha^{2}+\frac{2 \gamma}{\nu} K \alpha\right)^{-1 / 2} \frac{\min _{\Gamma} V_{1}^{0}}{\sqrt{2} \max _{\Gamma}\left|V^{0}\right|} \\
& \times \exp \left\{-\frac{2 c_{0} \sqrt{a b}}{\min _{\Gamma} V_{1}^{0}}\left(\left\|V^{0}\right\|_{2,2}+c_{2}\left\|\Delta V^{0}\right\|_{2}\right\},\right.
\end{aligned}
$$

где

$$
\begin{gathered}
\alpha=\frac{4 c_{0} c_{2}}{\nu} \sqrt{a b} \frac{\left\|\nabla V^{0}\right\|_{2}}{\min _{\Gamma} V_{1}^{0}}, \quad c_{0} \leqslant \max \left(8, \frac{11}{b}\right), \\
c_{2} \leqslant\left(1+\gamma^{2}+\gamma^{4}\right)^{1 / 2}, \quad \gamma=\min (a, b), \quad K \leqslant \frac{1}{2}\left(1+\sqrt{1+\frac{4 b}{e \sqrt{2}}}\right) .
\end{gathered}
$$

Возвращаясь к задаче $(1),(2)$, из теоремы 1 согласно $(3),(5)$ получаем, что при вьполнении условий $V^{0} \in W_{p}^{2}(\Omega)(p>2), \rho_{0} \in C_{\delta}\left(\Gamma_{1}\right)$ и (33) существует решение $(\rho, V)$ задачи (1), (2), причем $\rho \in C_{\delta^{\prime}}(\bar{\Omega}), V \in W_{p}^{2}(\Omega)$.

6. Существование решений задачи (1) в классах Гёльдера. Рассмотрим задачу Дирихле

$$
\Delta V=F, \quad x \in \Omega,\left.\quad V\right|_{\Gamma}=V^{0}
$$

где $F \in C_{\delta}(\bar{\Omega})(0<\delta<1)$, а функция $V^{0}$ на $\Gamma$ удовлетворяет условиям

$$
V^{0} \in C(\Gamma),\left.\quad V^{0}\right|_{\Gamma_{j}} \in C_{\delta}^{2}\left(\Gamma_{j}\right), \quad j=\overline{1,4}
$$

Известно [8], что для сушествования в $C_{\delta}^{2}(\bar{\Omega})$ решения задачи $(34)$ необходимо и достаточно, чтобы

$$
\Delta V^{0}\left(s_{j}\right)=F\left(s_{j}\right), \quad j=\overline{1,4}
$$

Здесь $s_{j}$ - вершины прямоугольника $\Omega$, занумерованные в порядке положительного обхода на $\Gamma, s_{1}=(0, b)$. Отсюда, в частности, вытекает, что функция $V^{0}$, удовлетворяющая условиям (35) и

$$
\Delta V^{0}\left(s_{j}\right)=0, \quad j=\overline{1,4}
$$

однозначно продолжается до гармонической в $\Omega$ функции из $C_{\delta}^{2}(\bar{\Omega})$. 
ТЕОрема 2. Пусть вектор-функиия $V^{0}$ удовлетворяет условиям (35), (37) и, следовательно, однозначно продолжсается в $\Omega$ до функиии $V^{0} \in C_{\delta}^{2}(\bar{\Omega}), \Delta V^{0}=0$ $($ в $\Omega)$. Пусть, кроме того, для функиий $\rho_{0} \in C_{\delta}^{1}\left(\Gamma_{1}\right)$ и $V^{0}$ выполнены условия (2), (33) и условия согласования

$$
\begin{gathered}
\rho_{0}\left(s_{j}\right) \frac{\partial V_{1}^{0}}{\partial x_{1}}\left(s_{j}\right)=0, \quad j=1,2, \\
\rho_{0}\left(s_{2}\right) \frac{\partial V_{1}^{0}}{\partial x_{1}}\left(s_{3}\right)=\rho_{0}\left(s_{1}\right) \frac{\partial V_{1}^{0}}{\partial x_{1}}\left(s_{4}\right)=0 .
\end{gathered}
$$

Тогда существует классическое решение $(\rho, V)$ задачи $(1)$ такое, что $\rho \in C_{\delta}^{1}(\bar{\Omega})$, $V \in C_{\delta}^{2}(\bar{\Omega})$.

ДокАЗАтельство. Рассмотрим задачу (4), (6), где $\varphi_{0}$ определено равенством (5). Поскольку $\varphi_{0} \in C_{\delta}^{2}\left(\Gamma_{1}\right), V^{0} \in C_{\delta}^{2}(\bar{\Omega})$, то из теоремы 1 вытекает существование решения $(\varphi, V)$ задачи $(4),(6)$, причем $(\varphi, V) \in C_{\beta}^{1}(\bar{\Omega}) \times C_{\beta}^{1}(\bar{\Omega})(0<\beta<1$ любое). Перепишем задачу (19) для вектор-функции $V$ в виде $(34)$, где $F=-\frac{1}{\nu}[\varphi, V] \in C_{\beta}(\bar{\Omega})$. Покажем, что для задачи $(34)$ с данным $F$ вьполнены условия $(36)$, которые в силу $\Delta V^{0}\left(s_{j}\right)=0$ $(j=1,2)$ сводятся к равенствам $F\left(s_{j}\right)=0(j=\overline{1,4})$.

Так как $\left.V_{2}\right|_{\Gamma_{2} \cup \Gamma_{4}}=0$, то

$$
\frac{\partial V_{2}}{\partial x_{1}}\left(s_{j}\right)=0 \quad \text { и } \quad f\left(s_{j}\right)=0,
$$

где $f=V_{2} / V_{1}$. Поэтому

$$
\frac{\partial \varphi}{\partial x_{1}}\left(s_{j}\right)=-f\left(s_{j}\right) \frac{\partial \varphi}{\partial x_{2}}\left(s_{j}\right)=0, \quad j=\overline{1,4} .
$$

Отсюда вытекает, что $F_{2}\left(s_{j}\right)=0(j=\overline{1,4})$. Далее, из равенств $(5),(15)-(17)$ и условий согласования (38) получаем

$$
\begin{gathered}
F_{1}\left(s_{j}\right)=\frac{1}{\nu} \frac{\partial \varphi}{\partial x_{2}}\left(s_{j}\right) \frac{\partial V_{1}}{\partial x_{1}}\left(s_{j}\right)=\frac{1}{\nu} \rho_{0}\left(s_{j}\right) V_{1}^{0}\left(s_{j}\right) \frac{\partial V_{1}^{0}}{\partial x_{1}}\left(s_{j}\right)=0, \quad j=1,2, \\
F_{1}\left(s_{3}\right)=\frac{1}{\nu} \rho_{0}\left(s_{2}\right) V_{1}^{0}\left(s_{2}\right) \frac{\partial V_{1}^{0}}{\partial x_{1}}\left(s_{3}\right) \exp \left\{-\int_{\Gamma_{2}} \frac{\partial f}{\partial x_{2}} d s\right\}=0 \\
F_{1}\left(s_{4}\right)=\frac{1}{\nu} \rho_{0}\left(s_{1}\right) V_{1}^{0}\left(s_{1}\right) \frac{\partial V_{1}^{0}}{\partial x_{1}}\left(s_{4}\right) \exp \left\{-\int_{\Gamma_{4}} \frac{\partial f}{\partial x_{2}} d s\right\}=0 .
\end{gathered}
$$

Итак, условия (36) вьполнены. Следовательно, из однозначной разрешимости задачи (34) получаем $V \in C_{\delta}^{2}(\bar{\Omega})$. Отсюда следует, что $f \in C_{\delta}^{2}(\bar{\Omega})$. Используя это обстоятельство, из (8), (15) заключаем, что $\xi_{V}(0, x) \in C_{\delta}^{2}(\bar{\Omega})$. Последнее влечет, что $\varphi(x)=\varphi_{0}\left(\xi_{V}(0, x)\right) \in C_{\delta}^{2}(\bar{\Omega})$ в силу соответствующей гладкости $\rho_{0}, V^{0}$ на $\Gamma_{1}$. Следовательно,

$$
\rho=\frac{1}{V_{1}} \frac{\partial \varphi}{\partial x_{2}} \in C_{\delta}^{1}(\bar{\Omega}) .
$$


Теорема доказана.

В теореме 2 условие (33) на данные задачи можно конкретизировать, используя оценки для производных функции $V^{0}$, полученные в [8].

Пусть функции $\gamma_{j} \in C^{1}[0, a](j=1,2)$ являются характеристиками уравнения $\left(V^{0}\right.$, $\nabla \varphi)=0$ и $\gamma_{1}<\gamma_{2}$. Положим $\Omega_{1}=\left\{x \in \mathbb{R}^{2}: x_{1} \in(0, a), x_{2} \in\left(\gamma_{1}\left(x_{1}\right), \gamma_{2}\left(x_{2}\right)\right)\right\}$. Аналогично может быть исследована задача (1) в криволинейной области $\Omega_{1}$ с условием $\left.V_{1}^{0}\right|_{\Gamma}>0$.

\section{СПИСОК ЦИТИРОВАННОЙ ЛИТЕРАТУРЫ}

[1] Кажихов А. В. О краевых задачах для уравнений Бюргерса сжимаемой жидкости в областях с подвижными границами // Динамика сплошной среды. Вып. 26. Новосибирск, 1976. C. $60-76$.

[2] Белов С. Я. Разрешимость вцелом задачи протекания для уравнений Бюргерса сжимаемой жидкости // Динамика сплошной среды. Вьп. 50. Новосибирск, 1981. С. 3-14.

[3] Антонцев С. Н., Кажихов А. В., Монахов В. Н. Краевые задачи механики неоднородных жидкостей. Новосибирск: Наука, 1983.

[4] Ладыженская О. А., Уральцева Н. Н. Линейные и квазилинейные уравнения эллиптического типа. М.: Наука, 1964.

[5] Петровский И.Г. Лекции по теории обыкновенных дифференциальных уравнений. М.: МГУ, 1984.

[6] Ильин В.П. К теоремам вложения // Тр. МИАН. 1959. Т. 53. С. 359-386.

[7] Кошелев А.И. Априорные оценки в $L_{p}$ и обобщенные решения эллиптических уравнений и систем // УМН. 1958. Т. 13. № 4. С. 29-88.

[8] Волков Е. А. О дифференциальных свойствах решений краевых задач для уравнений Лапласа и Пуассона на прямоугольнике // Тр. МИАН. 1965. Т. 77. С. 87-112.

Дальневосточный государственный университет, г. Владивосток

Поступило 21.03 .95

Исправленный вариант

27.02 .96 